\title{
Structure-preserving Model Reduction of Hamiltonian Systems for Linear Elasticity
}

\author{
Patrick Buchfink* \\ * Institute of Applied Analysis and Numerical Simulation, University \\ of Stuttgart, Pfaffenwaldring 57, 70569 Stuttgart, Germany.
}

Keywords: Symplectic model reduction, linear elasticity, Hamiltonian system, Proper Symplectic Decomposition.

\section{INTRODUCTION}

The goal of this paper is to apply model reduction techniques that preserve the Hamiltonian structure (see e.g. Maboudi Afkham and Hesthaven (2016)) to a highdimensional, linear elasticity model. The Hamiltonian structure of a linear elasticity model is presented and the structure-preserving model reduction is introduced. Further, two structure-preserving model reduction techniques are compared to a technique that does not preserve the Hamiltonian structure in a numerical experiment.

\section{HIGH-DIMENSIONAL MODEL}

\subsection{Second-order formulation}

The model for two-dimensional linear elasticity is derived from a geometrically linear, small strain formulation. The Lamé constants $\lambda_{\mathrm{L}}, \mu_{\mathrm{L}} \in \mathbb{R}$ with $\mu_{\mathrm{L}}>0,3 \lambda_{\mathrm{L}}+2 \mu_{\mathrm{L}}>0$ are used to describe the linear elastic material behavior. Additionally, nondimensionalization is applied. This results in a dimensionless Lamé-Navier initial boundary value problem. Application of the Finite Element Method (FEM) with a triangular grid and linear shape functions yields the initial value problem of size $n \in \mathbb{N}$ which describes the evolution of the unknown, parameter-dependent, displacement field $\boldsymbol{q}:\left[t_{0}, t_{\mathrm{e}}\right] \times \mathcal{P} \rightarrow \mathbb{R}^{n}$ with

$$
\boldsymbol{M} \frac{\mathrm{d}^{2}}{\mathrm{~d} t^{2}} \boldsymbol{q}(t, \boldsymbol{\mu})+\boldsymbol{K}(\boldsymbol{\mu}) \boldsymbol{q}(t, \boldsymbol{\mu})=\boldsymbol{f}(t),
$$

with the initial value conditions

$$
\boldsymbol{q}\left(t_{0}, \boldsymbol{\mu}\right)=\boldsymbol{q}_{0}, \quad \frac{\mathrm{d}}{\mathrm{d} t} \boldsymbol{q}\left(t_{0}, \boldsymbol{\mu}\right)=\boldsymbol{v}_{0}
$$

where $\boldsymbol{\mu}=\left[\lambda_{\mathrm{L}}, \mu_{\mathrm{L}}\right]^{\top} \in \mathcal{P}$ are the parameters from the parameter space $\mathcal{P} \subset \mathbb{R}^{2}, \boldsymbol{M} \in \mathbb{R}^{n \times n}$ is the mass matrix, $\boldsymbol{K}(\boldsymbol{\mu}) \in \mathbb{R}^{n \times n}$ is the parameter-dependent stiffness matrix, $\boldsymbol{f}(t)$ is the force vector, $\boldsymbol{q}_{0} \in \mathbb{R}^{n}$ is the initial displacement, and $\boldsymbol{v}_{0} \in \mathbb{R}^{n}$ is the initial velocity at the initial time $t_{0} \in \mathbb{R}$. The final time is denoted with $t_{\mathrm{e}} \in \mathbb{R}$. The parameters $\boldsymbol{\mu}$ are assumed fixed during a single simulation. The parameter-dependence is only denoted if required in the following which is why we abbreviate $\boldsymbol{K}=\boldsymbol{K}(\boldsymbol{\mu})$, $\boldsymbol{q}(t)=\boldsymbol{q}(t, \boldsymbol{\mu})$.

\subsection{Hamiltonian formulation}

In order to derive a Hamiltonian formulation of the system, the force vector $\boldsymbol{f}(t)=\boldsymbol{f}$ has to be constant. The second- order system (1) is rewritten in terms of a first-order system with the linear momentum $\boldsymbol{p}(t)=\boldsymbol{M} \frac{\mathrm{d}}{\mathrm{d} t} \boldsymbol{q}(t)$ and the state vector $\boldsymbol{x}(t)=\left[\boldsymbol{q}^{\boldsymbol{\top}}(t), \boldsymbol{p}^{\top}(t)\right]^{\boldsymbol{\top}}$. The corresponding Hamilton's equation reads

$$
\begin{aligned}
\frac{\mathrm{d}}{\mathrm{d} t} \boldsymbol{x}(t) & =\mathbb{J}_{2 n} \nabla_{\boldsymbol{x}} \mathcal{H}(\boldsymbol{x}(t))=\mathbb{J}_{2 n}(\boldsymbol{H} \boldsymbol{x}(t)+\boldsymbol{h}), \\
\left.\boldsymbol{x}\left(t_{0}\right)\right) & =\boldsymbol{x}_{0}=\left[\boldsymbol{q}_{0}^{\top}, \boldsymbol{v}_{0}^{\top}\right]^{\top}
\end{aligned}
$$

with the definitions

$$
\mathbb{J}_{2 n}=\left[\begin{array}{cc}
\mathbf{0}_{n} & \boldsymbol{I}_{n} \\
-\boldsymbol{I}_{n} & \mathbf{0}_{n}
\end{array}\right], \quad \boldsymbol{H}=\left[\begin{array}{cc}
\boldsymbol{K} & \mathbf{0}_{n} \\
\mathbf{0}_{n} & \boldsymbol{M}^{-1}
\end{array}\right], \quad \boldsymbol{h}=\left[\begin{array}{c}
-\boldsymbol{f} \\
\mathbf{0}_{n \times 1}
\end{array}\right]
$$

and the corresponding Hamiltonian function

$$
\mathcal{H}(\boldsymbol{x})=1 / 2 \boldsymbol{x}^{\top} \boldsymbol{H} \boldsymbol{x}+\boldsymbol{x}^{\top} \boldsymbol{h}
$$

where $\mathbf{0}_{n} \in \mathbb{R}^{n \times n}$ is the matrix and $\mathbf{0}_{n \times 1} \in \mathbb{R}^{n}$ the vector of all zeros and $\boldsymbol{I}_{n} \in \mathbb{R}^{n \times n}$ is the identity matrix.

An essential feature of the definition of Hamilton's equation is that the Hamiltonian function is preserved along the solution, i.e. $\frac{\mathrm{d}}{\mathrm{d} t} \mathcal{H}(\boldsymbol{x}(t))=0$, where $\boldsymbol{x}(t)$ is the solution of the Hamiltonian system (3).

\section{STRUCTURE-PRESERVING MODEL REDUCTION}

\subsection{Motivation}

The size $2 n$ of the system (3) is in general high which is why the model is called high-dimensional model. Multiquery scenarios or real-time application require model reduction techniques. The goal of the structure-preserving model reduction is to derive a reduced system that preserves the Hamiltonian structure of the system (3). To this end, the full state vector $\boldsymbol{x} \in \mathbb{R}^{2 n}$ is approximated with a low-dimensional state $\widehat{\boldsymbol{x}} \in \mathbb{R}^{2 k}$ of size $2 k$ where $k \in \mathbb{N}$ and $k \ll n$. A reduced order basis (ROB) $\boldsymbol{V} \in \mathbb{R}^{2 n \times 2 k}$ is used for the approximation

$$
\boldsymbol{x}(t) \approx \boldsymbol{V} \widehat{\boldsymbol{x}}(t) .
$$

The ROB is chosen to be symplectic matrix, i.e.

$$
\boldsymbol{V}^{\top} \mathbb{J}_{2 n} \boldsymbol{V}=\mathbb{J}_{2 k} \text {. }
$$

If the ROB is symplectic, the existence of its symplectic inverse $\boldsymbol{V}^{+}=\mathbb{J}_{2 k}^{\top} \boldsymbol{V}^{\top} \mathbb{J}_{2 n} \in \mathbb{R}^{2 k \times 2 n}$ with $\boldsymbol{V}^{+} \boldsymbol{V}=\boldsymbol{I}_{2 k}$ is guaranteed.

The approximation (7) is inserted into Hamilton's equation (3) while the system is projected with $\boldsymbol{W}^{\top}=\boldsymbol{V}^{+}$. 
This yields the low-dimensional initial value problem as the reduced Hamiltonian's equation

$$
\begin{aligned}
\frac{\mathrm{d}}{\mathrm{d} t} \widehat{\boldsymbol{x}}(t) & =\mathbb{J}_{2 k} \nabla_{\widehat{\boldsymbol{x}}} \widehat{\mathcal{H}}(\widehat{\boldsymbol{x}}(t))=\mathbb{J}_{2 k}(\widehat{\boldsymbol{H}} \widehat{\boldsymbol{x}}(t)+\widehat{\boldsymbol{h}}), \\
\widehat{\boldsymbol{x}}\left(t_{0}\right) & =\boldsymbol{W}^{\top} \boldsymbol{x}_{0}=\boldsymbol{V}^{+} \boldsymbol{x}_{0}
\end{aligned}
$$

with the definitions

$$
\widehat{\boldsymbol{H}}=\boldsymbol{V}^{\top} \boldsymbol{H} \boldsymbol{V}, \quad \widehat{\boldsymbol{h}}=\boldsymbol{V}^{\top} \boldsymbol{h}
$$

with the corresponding reduced Hamiltonian function

$$
\widehat{\mathcal{H}}(\widehat{\boldsymbol{x}})=1 / 2 \widehat{\boldsymbol{x}}^{\top} \widehat{\boldsymbol{H}} \widehat{\boldsymbol{x}}+\widehat{\boldsymbol{x}}^{\top} \widehat{\boldsymbol{h}} .
$$

\subsection{Snapshot-based basis generation}

A snapshot-based basis generation is used. This means several simulations with the high-dimensional model (3) are used to provide $n_{\mathrm{s}} \in \mathbb{N}$ state vectors $\boldsymbol{x}_{i}^{\mathrm{s}}=\boldsymbol{x}\left(t_{i}, \boldsymbol{\mu}_{i}\right)$, $i=1, \ldots, n_{\mathrm{s}}$ which are referred to as snapshots. The ROB is computed based on the ensemble of all snapshots, e.g. with the snapshot matrix $\boldsymbol{X}^{\mathrm{s}}=\left[\boldsymbol{x}_{1}^{\mathrm{s}}, \ldots, \boldsymbol{x}_{n_{\mathrm{s}}}^{\mathrm{s}}\right] \in \mathbb{R}^{2 n \times n_{\mathrm{s}}}$.

The Proper Symplectic Decomposition (PSD) (see Peng and Mohseni (2016)) chooses the ROB to minimize the residual of the symplectic projection $\left(\boldsymbol{I}-\boldsymbol{V} \boldsymbol{V}^{+}\right) \boldsymbol{X}^{\mathrm{s}}$ of the snapshot matrix, i.e.

$$
\begin{aligned}
& \underset{\boldsymbol{V} \in \mathbb{R}^{2 n \times 2 k}}{\operatorname{minimize}}\left\|\left(\boldsymbol{I}-\boldsymbol{V} \boldsymbol{V}^{+}\right) \boldsymbol{X}^{\mathrm{s}}\right\|_{F}, \\
& \text { subject to } \quad \boldsymbol{V}^{\top} \mathbb{J}_{2 n} \boldsymbol{V}=\mathbb{J}_{2 k},
\end{aligned}
$$

while the side condition (14) requires the ROB to be symplectic (cf. (8)). An explicit solution for the minimization problem (13)-(14) is yet not known and can only be provided under restrictive assumptions on the structure of the ROB. Peng and Mohseni (2016) for example present the Cotangent Lift and the Complex SVD whereas Maboudi Afkham and Hesthaven (2016) present a greedy procedure to solve the minimization problem.

\section{NUMERICAL RESULTS}

In this section, two structure-preserving model reduction techniques are compared with a technique that does not preserve the Hamiltonian structure. A relative, mean over time, state space error measure is considered to compare the model reduction techniques numerically

$$
e(2 k)=\sum_{i=1}^{n_{\mathrm{t}}} \frac{1}{n_{\mathrm{t}}} \frac{\left\|\boldsymbol{x}\left(t_{i}\right)-\boldsymbol{V} \widehat{\boldsymbol{x}}\left(t_{i}\right)\right\|_{2}}{\left\|\boldsymbol{x}\left(t_{i}\right)\right\|_{2}}
$$

where $t_{1}, \ldots, t_{n_{\mathrm{t}}} \in\left[t_{0}, t_{\mathrm{e}}\right]$ are the discrete times and the variable $2 k$ is the size of the ROB $\boldsymbol{V}$.

The example scenario is a beam that is clamped on one side and loaded with a force on the other side. The force acts on the boundary and pushes orthogonal to the beam. The finite element model has $2 n=1152$ degrees of freedom. The ROB is created from snapshots computed with one simulation with $\boldsymbol{\mu}_{1}=\left[1.2110^{11} \mathrm{~N} / \mathrm{m}, 8.0810^{10} \mathrm{~N} / \mathrm{m}\right]^{\top}$. The reduction techniques are compared for the same parameter $\boldsymbol{\mu}_{1}$. In all cases, a symplectic integration scheme, namely the implicit midpoint rule, is used for time integration.

The Cotangent Lift and the greedy approach from Maboudi Afkham and Hesthaven (2016) are considered as structure-preserving model reduction techniques in the following. Both are compared with the Proper Orthogonal

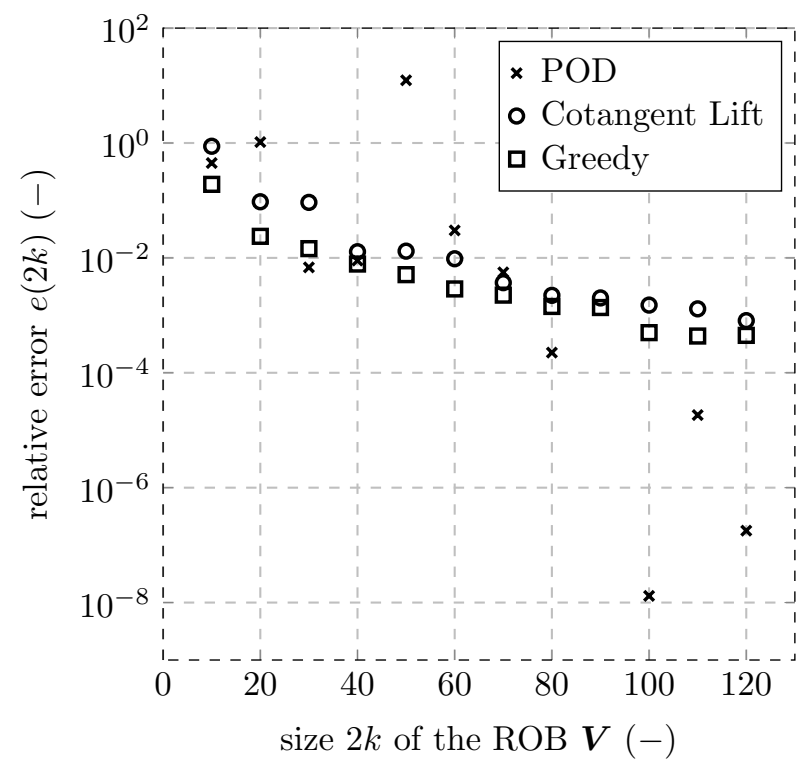

Fig. 1. Relative, mean over time, state space error $e(2 k)$ for different ROB sizes $2 k$ in a semi-logarithmic plot.

Decomposition (POD) which does not necessarily preserve the Hamiltonian structure. For more details on the POD, see e.g. Volkwein (2013).

The results of the comparison are displayed in Figure 1 in terms of the error measure from (15). It shows that the POD is able to compute the best ROB with $2 k=100$. But the POD also yields the highest error with $e(90)>10^{2}$ which was excluded from Figure 1 for the sake of a better overview. Overall, the results of both structure-preserving PSD techniques show less peaks and thus are more reliable. The greedy approach is in all considered cases better than the Cotangent Lift.

\section{CONCLUSION}

The structure-preserving model reduction was introduced and the Proper Symplectic Decomposition was presented as corresponding snapshot-based model reduction technique. The numerical results showed that the considered structure-preserving model reduction techniques provide bases that are more reliable than the ones created with the Proper Orthogonal Decomposition.

\section{ACKNOWLEDGEMENTS}

The author expresses a special thanks to the supervisors of his master thesis (A. Bhatt, B. Haasdonk, and J. Fehr).

\section{REFERENCES}

Maboudi Afkham, B. and Hesthaven, J.S. (2016). Structure Preserving Model Reduction of Parametric Hamiltonian Systems.

Peng, L. and Mohseni, K. (2016). Symplectic Model Reduction of Hamiltonian Systems. Siam Journal on Scientific Computing, 38, A1-A27.

Volkwein, S. (2013). Proper Orthogonal Decomposition: Theory and Reduced-Order Modelling. Lecture notes, Universität Konstanz. 